\title{
Study of Structure and Electronic Properties of
}

\section{Hetero-Interfaces for Photovoltaic Applications}

\author{
Rabi Khanal, ${ }^{*} \dagger$ Nicholas Ayers, ${ }^{\dagger}$ Sheila Briggs, ${ }^{\dagger}$ Yi Wang, ${ }^{\ddagger}$ Soumik
} Banerjee, "and Samrat Choudhury, ${ }^{* \dagger}$

$\dagger$ Department of Chemical and Materials Engineering, University of Idaho, Moscow, ID 83844, USA

$\ddagger$ Department of Chemical and Materials Engineering, Pennsylvania State University, State College, University Park, PA 16802, USA

ฯ School of Mechanical and Materials Engineering, Washington State University, Pullman, WA, 99164, USA

E-mail: rkhanal@uidaho.edu; samrat@uidaho.edu 

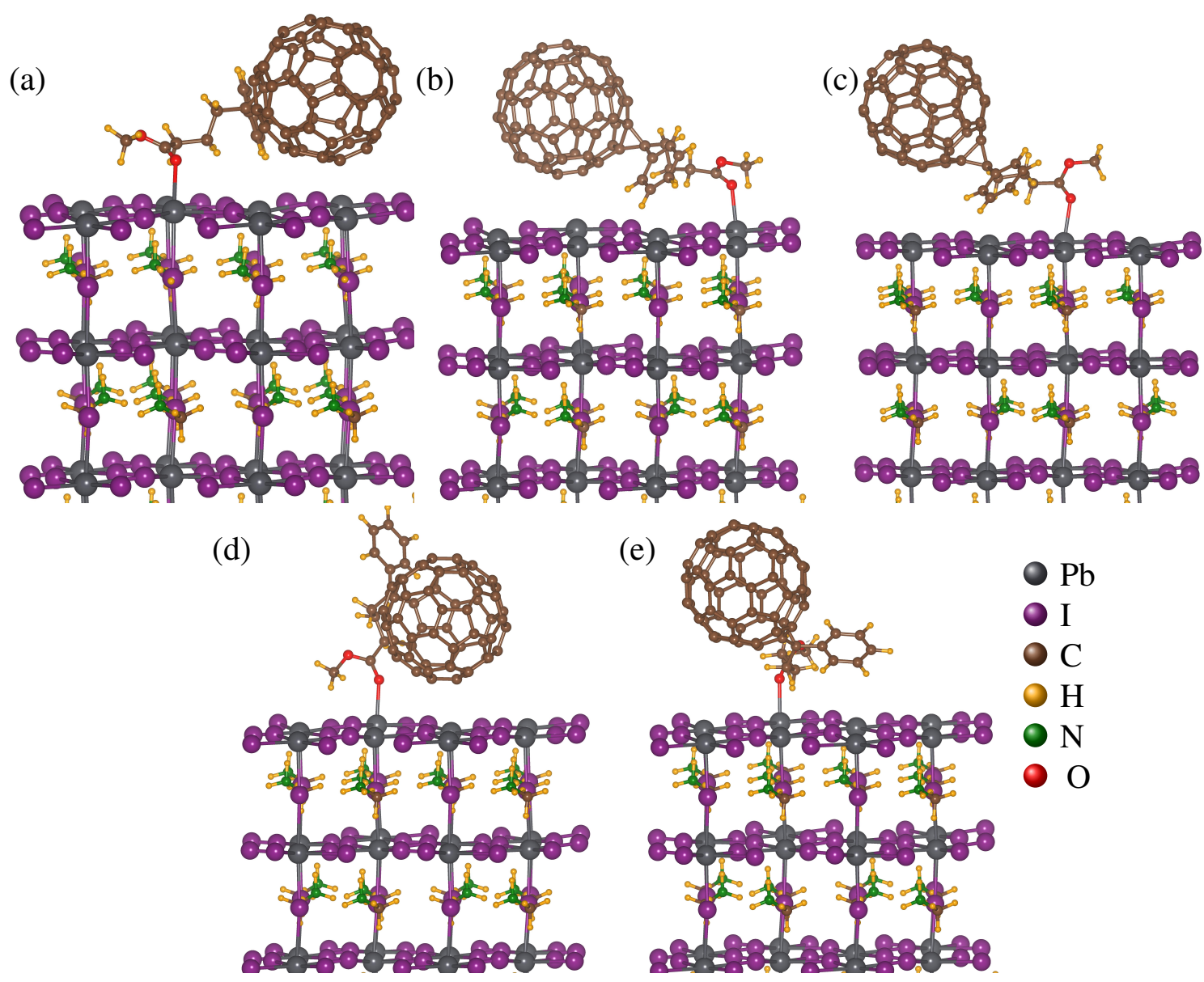

(e)

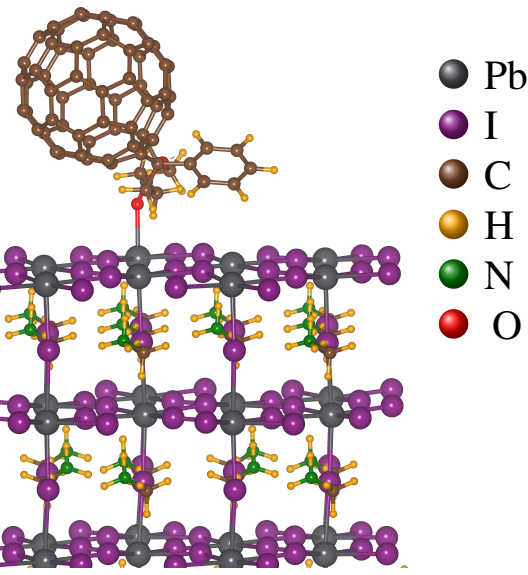

Figure S1: Atomic structure of $\mathrm{PCBM}$ /perovskite interfaces with perovskite $\mathrm{PbI}_{2}$ surface termination. Different structure corresponds to the minimum or close to minimum energy structures as shown in the Table S1.

Table S1: Perovskite/PCBM interface with $\mathrm{PbI}_{2}$ surface termination: inter-atomic distances for lowest five energy configurations, number in parentheses represents the total number of distances less than $3.5 \AA$.

\begin{tabular}{|l|l|l|l|l|l|l|}
\hline Model & Energy $(\mathrm{eV})$ & $\mathrm{Pb}-\mathrm{O}_{1}(\AA)$ & $\mathrm{Pb}-\mathrm{O}_{2}(\AA)$ & $\mathrm{I}-\mathrm{H}(\AA)$ & $\mathrm{Pb}_{60}(\AA)$ & $\mathrm{Pb}-\mathrm{C}(\AA)$ \\
\hline (a) & 0 & 2.68 & 4.41 & $3.02(5)$ & $3.01(2)$ & 3.66 \\
$(\mathrm{~b})$ & 0.12 & 2.55 & 4.39 & $2.95(6)$ & $3.77(0)$ & 3.39 \\
$(\mathrm{c})$ & 0.34 & 2.54 & 4.65 & $3.15(8)$ & $5.06(0)$ & 3.34 \\
$(\mathrm{~d})$ & 0.49 & 2.60 & 4.33 & $2.92(2)$ & $3.16(2)$ & 3.72 \\
(e) & 0.62 & 2.51 & 4.57 & $2.99(4)$ & $5.58(0)$ & 3.63 \\
\hline
\end{tabular}




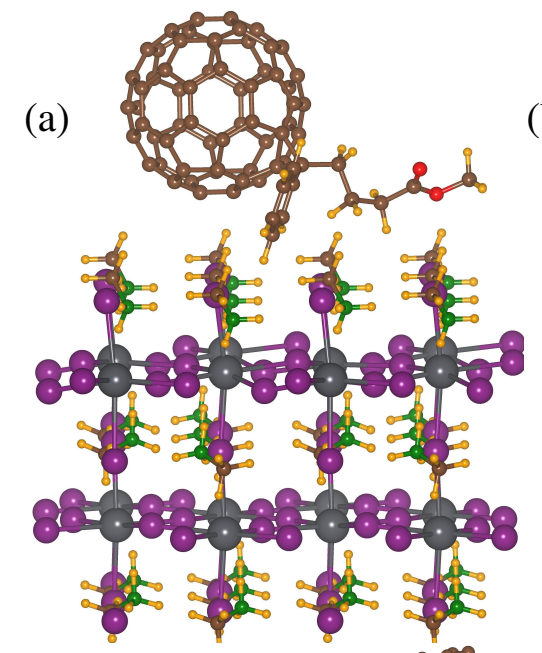

(b)

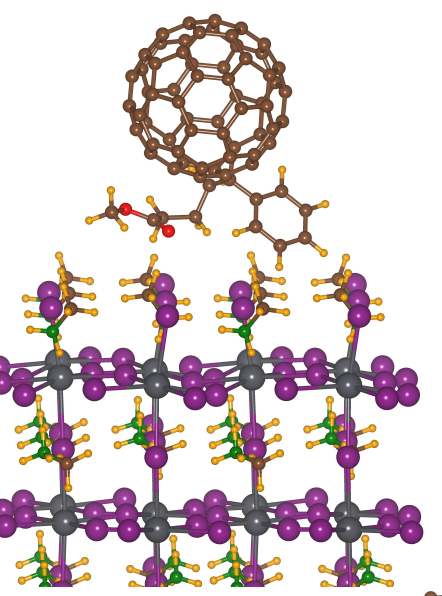

(d)
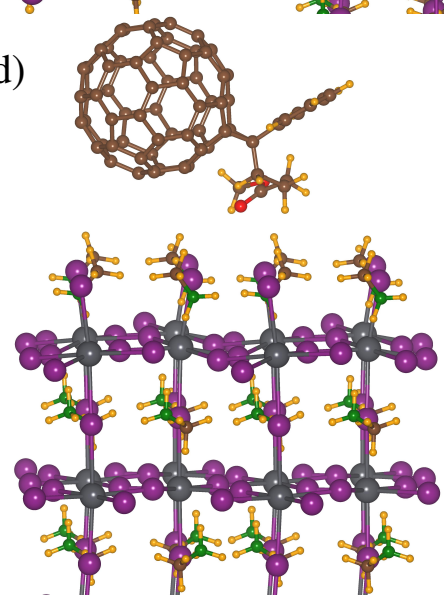

(c)

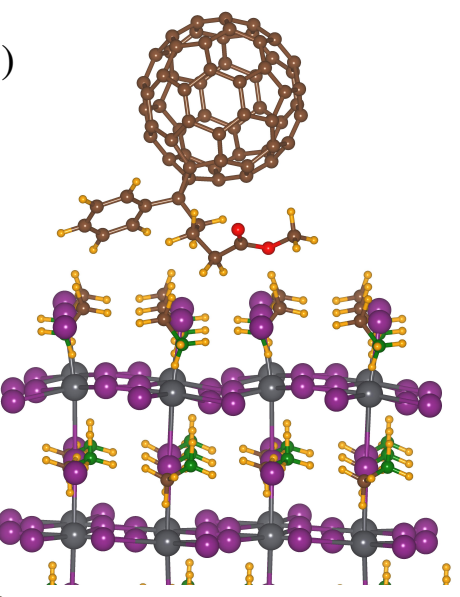

(e)

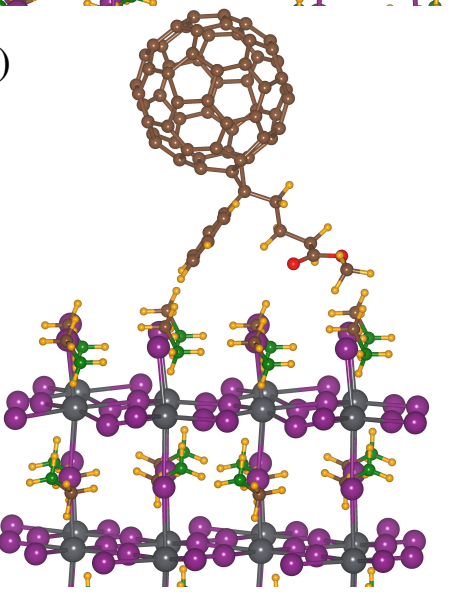

Figure S2: Atomic structure of PCBM/perovskite interfaces with perovskite MAI surface termination. Different structure corresponds to the minimum or close to minimum energy structures as shown in the Table S2.

Table S2: Perovskite-PCBM interface with MAI surface termination:inter-atomic distances for lowest five energy configurations, number in parentheses represents the total number of distances less than $3.5 \AA$.

\begin{tabular}{|l|l|l|l|l|l|l|}
\hline Model & Energy $(\mathrm{eV})$ & $\mathrm{I}-\mathrm{H}(\AA)$ & $\mathrm{H}-\mathrm{C}(\AA)$ & $\mathrm{H}-\mathrm{O}_{1}(\AA)$ & $\mathrm{H}-\mathrm{O}_{2}(\AA)$ & $\mathrm{I}-\mathrm{C}(\AA)$ \\
\hline (a) & 0.00 & $2.92(3)$ & $3.05(5)$ & 3.41 & 2.96 & 3.71 \\
(b) & 0.26 & $2.91(3)$ & $3.02(3)$ & 2.27 & 3.68 & 3.83 \\
(c) & 0.30 & $3.17(2)$ & $3.07(4)$ & 4.26 & 2.38 & 3.87 \\
(d) & 0.34 & $3.08(2)$ & $2.81(8)$ & 3.07 & 2.66 & 4.14 \\
(e) & 0.35 & $3.07(3)$ & $2.85(3)$ & 2.34 & 4.24 & 3.76 \\
\hline
\end{tabular}




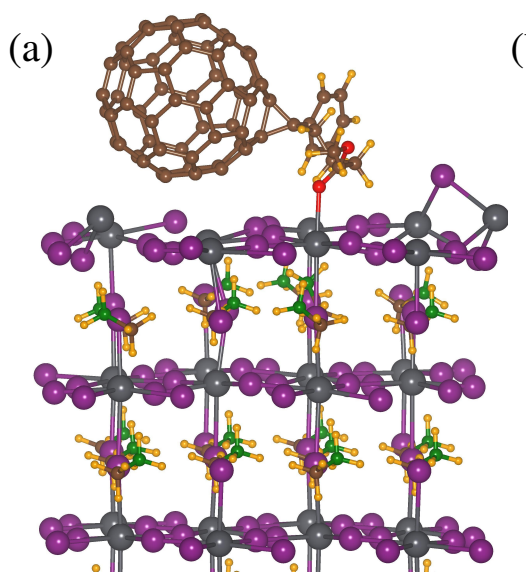

(b)

(d)

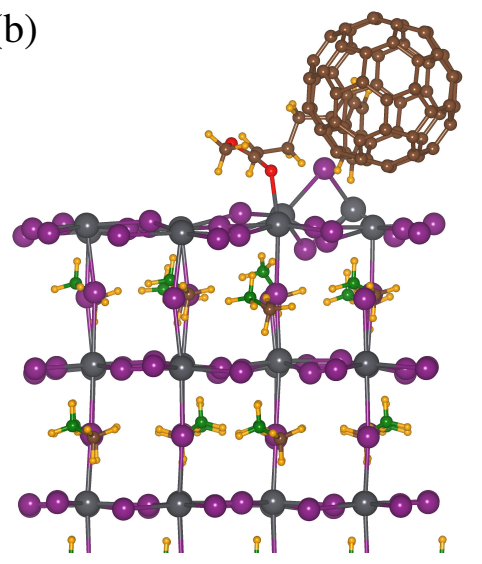

(e) (c)

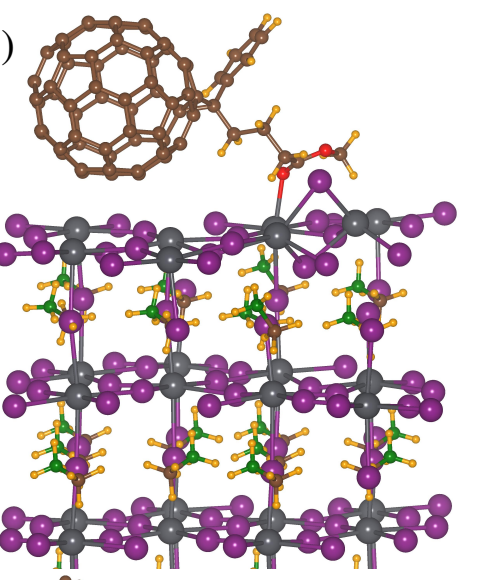

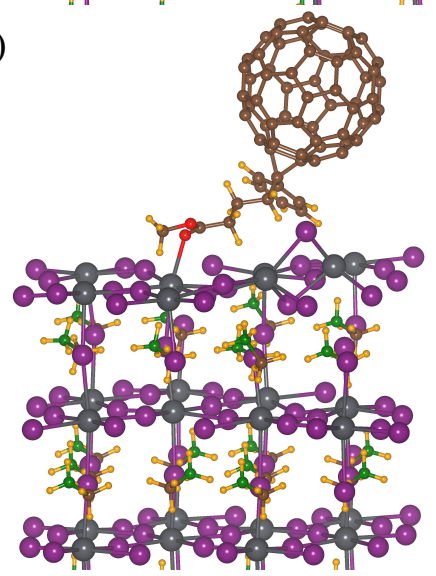

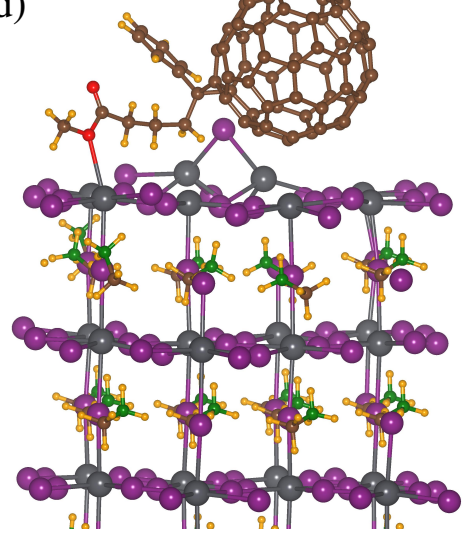

Figure S3: Atomic structure of PCBM/perovskite interfaces with iodine interstitial defects on the $\mathrm{PbI}_{2}$ surface termination. Different structure corresponds to the minimum or close to minimum energy structures as shown in the Table S3.

Table S3: Perovskite-PCBM interface (perovskite $\mathrm{PbI}_{2}$ surface termination) with I interstitial defects: inter-atomic distances for lowest five energy configurations, number in parentheses represents the total number of distances less than $3.5 \AA$.

\begin{tabular}{|l|l|l|l|l|l|l|}
\hline Model & Energy $(\mathrm{eV})$ & $\mathrm{Pb}^{-\mathrm{O}_{1}}(\AA)$ & $\mathrm{Pb}-\mathrm{O}_{2}(\AA)$ & $\mathrm{I}-\mathrm{H}(\AA)$ & $\mathrm{Pb}_{-60}(\AA)$ & $\mathrm{Pb}-\mathrm{C}(\mathrm{ester})(\AA)$ \\
\hline (a) & 0.00 & 2.51 & 4.52 & $3.12(7)$ & $3.39(3)$ & 3.50 \\
$(\mathrm{~b})$ & 0.09 & 2.61 & 4.45 & $3.06(5)$ & $3.38(2)$ & 3.63 \\
(c) & 0.31 & 2.53 & 4.31 & $3.09(3)$ & $3.27(1)$ & 3.43 \\
$(\mathrm{~d})$ & 0.58 & 5.15 & 2.98 & $2.89(6)$ & $3.05(1)$ & 3.83 \\
(e) & 0.72 & 2.64 & 4.16 & $3.04(7)$ & $5.34(0)$ & 3.50 \\
\hline
\end{tabular}


(a)

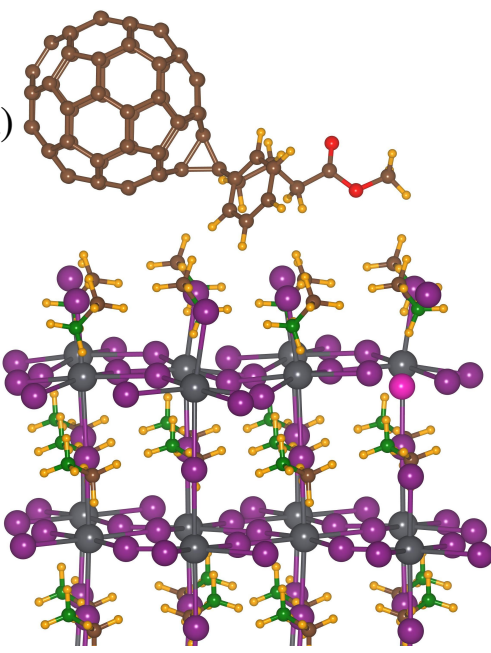

(b)
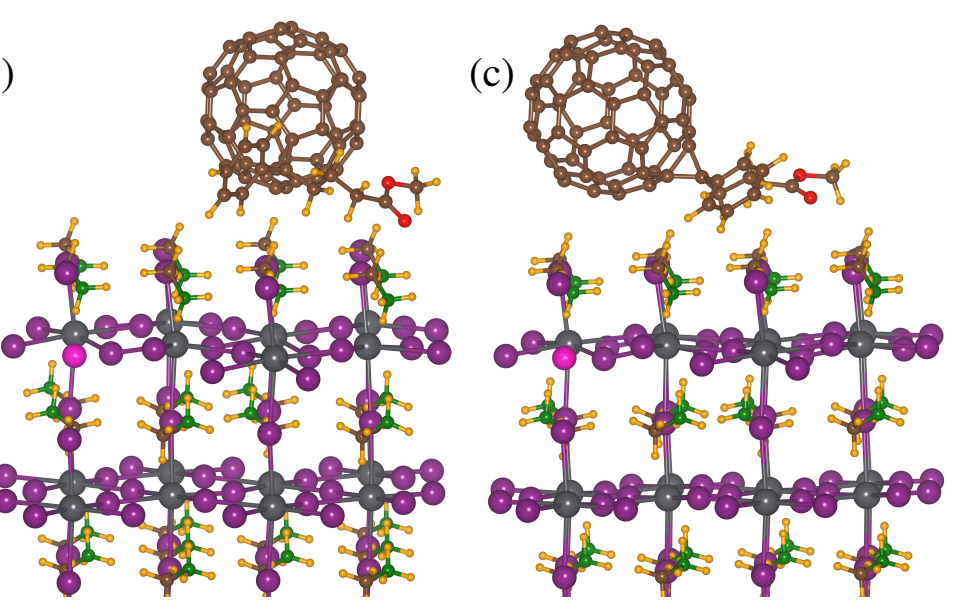

(d)

(e)
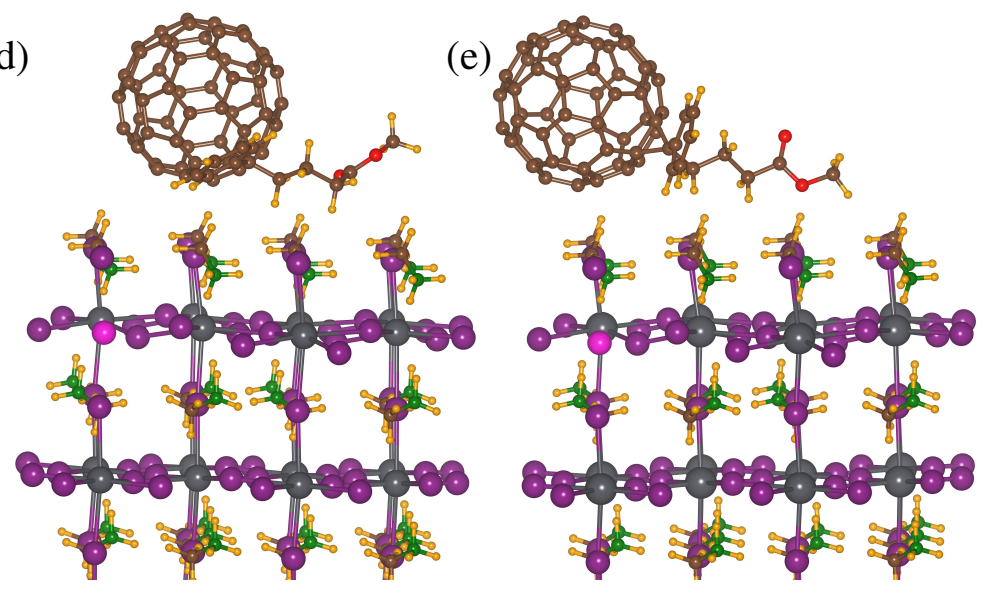

Figure S4: Atomic structure of PCBM/perovskite interfaces with iodine antisite defects on MAI surface terminations. Different structure corresponds to the minimum or close to minimum energy structure as shown in the Table S4. Pink atom represent defective iodine atom.

Table S4: Perovskite-PCBM interface (perovskite MAI surface termination) with I antisite: interatomic distances for lowest five energy configurations, number in parentheses represents the total number of distances less than $3.5 \AA$.

\begin{tabular}{|l|l|l|l|l|l|l|}
\hline Position & Energy $(\mathrm{eV})$ & $\mathrm{I}-\mathrm{H}(\AA)$ & $\mathrm{H}-\mathrm{C}(\AA)$ & $\mathrm{H}-\mathrm{O}_{1}(\AA)$ & $\mathrm{H}-\mathrm{O}_{2}(\AA)$ & $\mathrm{I}-\mathrm{C}(\AA)$ \\
\hline (a) & 0.00 & $3.68(0)$ & $2.74(15)$ & 2.81 & 4.42 & 3.53 \\
(b) & 0.01 & $2.80(4)$ & $2.98(10)$ & 4.89 & 3.23 & 3.73 \\
(c) & 0.48 & $3.15(2)$ & $2.48(14)$ & 4.31 & 2.18 & 3.55 \\
$(\mathrm{~d})$ & 0.50 & $2.93(3)$ & $2.57(18)$ & 2.99 & 3.84 & 3.84 \\
(e) & 0.78 & $3.52(0)$ & $2.30(21)$ & 2.72 & 4.55 & 3.51 \\
\hline
\end{tabular}




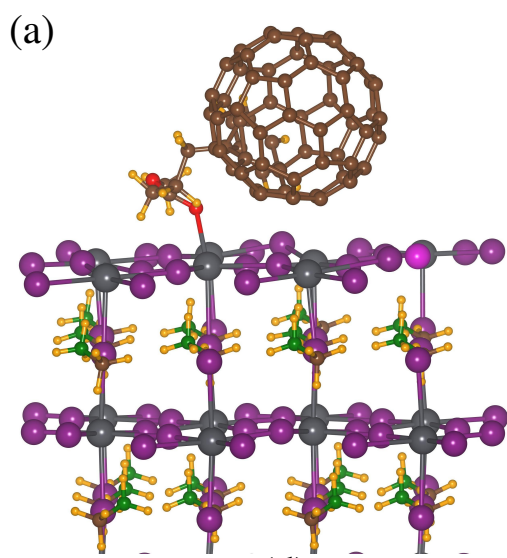

(d) (b)

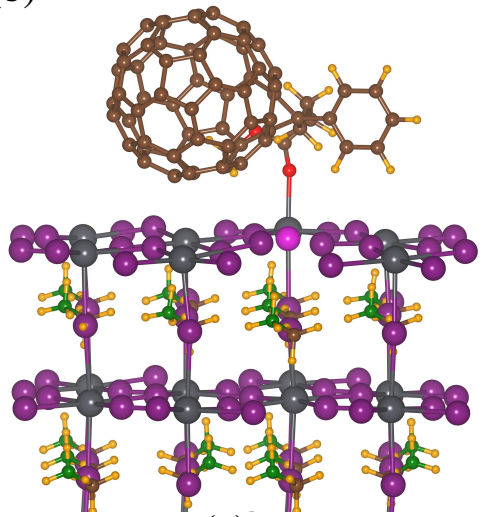

(e) (c)

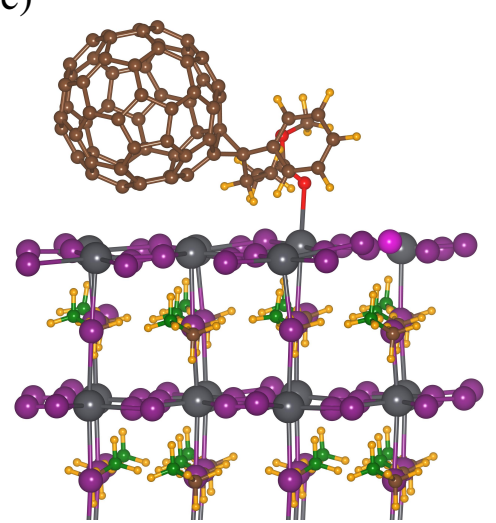

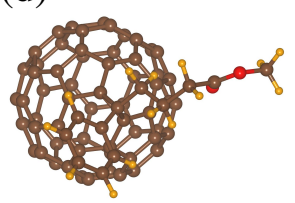
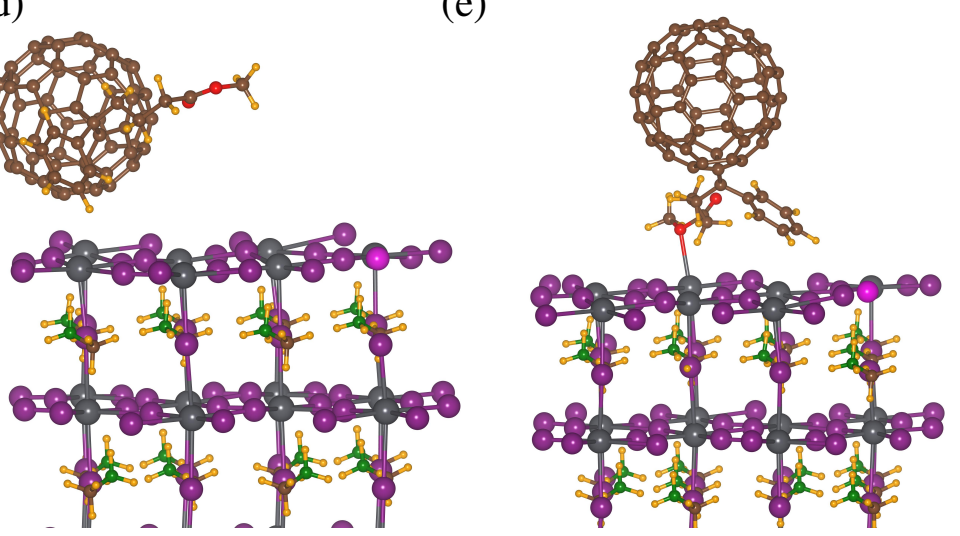

Figure S5: Atomic structure of PCBM/perovskite interfaces with iodine antisite defects on $\mathrm{Pb}_{2}$ surface termination. Different structure corresponds to the minimum or close to minimum energy structures as shown in the Table S5. Pink atom represent defective iodine atom.

Table S5: Perovskite-PCBM interface ( $\mathrm{PbI}_{2}$ surface termination) with I antisite defect: interatomic distances for lowest five energy configurations, number in parentheses represents the total number of distances less than $3.5 \AA$.

\begin{tabular}{|l|l|l|l|l|l|l|}
\hline Position & Energy $(\mathrm{eV})$ & $\mathrm{Pb}-\mathrm{O}_{1}(\AA)$ & $\mathrm{Pb}^{-} \mathrm{O}_{2}(\AA)$ & $\mathrm{I}-\mathrm{H}(\AA)$ & $\mathrm{Pb}^{-} \mathrm{C}_{60}(\AA)$ & $\mathrm{Pb}-\mathrm{C}(\mathrm{ester})(\AA)$ \\
\hline (a) & 0.00 & 2.56 & 4.48 & $3.06(8)$ & 3.25 & 3.40 \\
$(\mathrm{~b})$ & 0.08 & 2.56 & 4.48 & $3.23(3)$ & 3.17 & 3.75 \\
(c) & 0.17 & 2.88 & 4.92 & $2.93(7)$ & 3.40 & 3.59 \\
$(\mathrm{~d})$ & 0.54 & 8.52 & 8.48 & $3.65(0)$ & 3.04 & - \\
$(\mathrm{e})$ & 0.75 & 4.49 & 2.85 & $3.04(5)$ & - & 3.26 \\
\hline
\end{tabular}




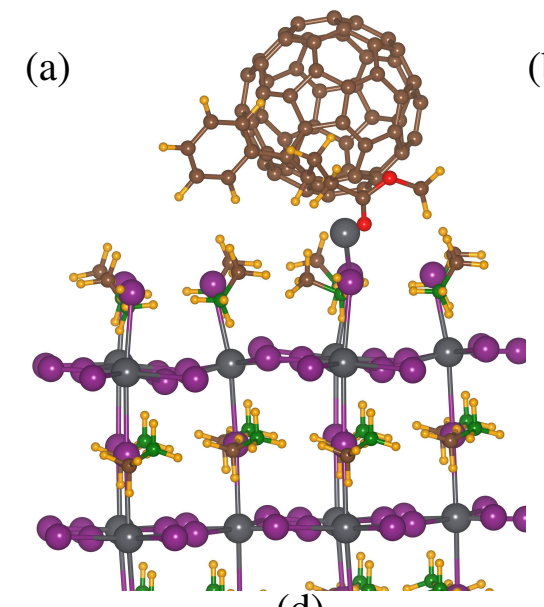

(d)

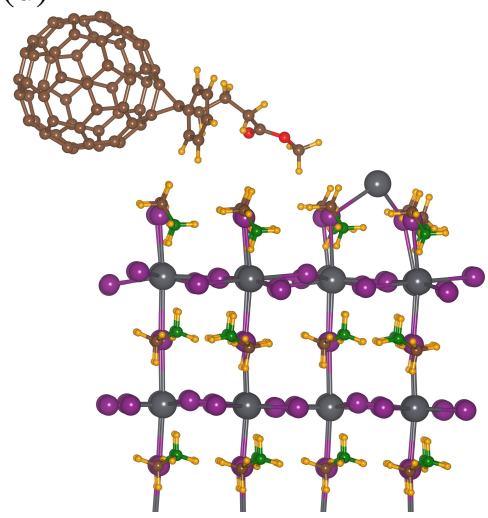

(b)

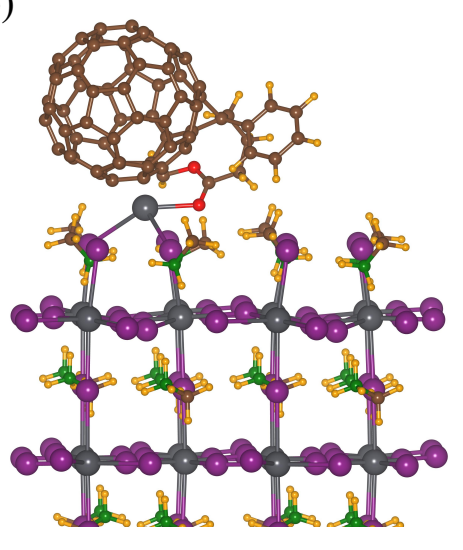

(c)

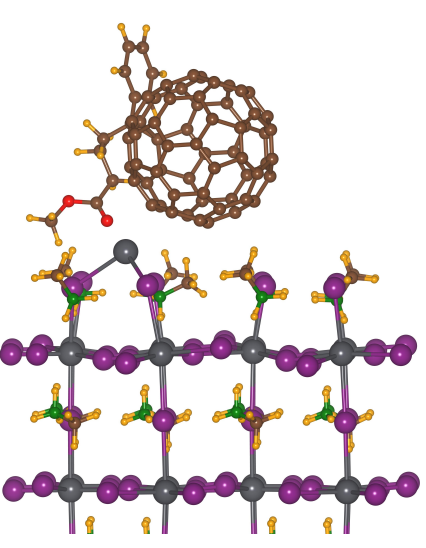

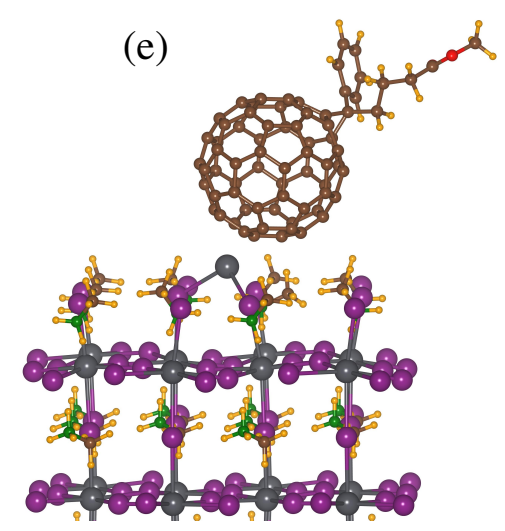

Figure S6: Atomic structure of PCBM/perovskite interfaces with $\mathrm{Pb}$ interstitials atom on MAI surface termination. Different structure corresponds to the minimum or close to minimum energy structures as shown in the Table S6.

Table S6: Perovskite-PCBM interface (MAI surface termination) with Pb interstitials; inter-atomic distances in $\AA$ for lowest five energy configurations, number in parentheses represents the total number of distances less than $3.5 \AA$.

\begin{tabular}{|l|l|l|l|l|l|l|l|}
\hline Position & Energy (eV) & $\mathrm{Pb}^{-\mathrm{O}_{1}}$ & $\mathrm{~Pb}-\mathrm{O}_{2}$ & $\mathrm{I}-\mathrm{H}$ & $\mathrm{H}-\mathrm{C}$ & $\mathrm{Pb}-\mathrm{C}(60)$ & $\mathrm{Pb}-\mathrm{C}(\mathrm{ester})$ \\
\hline (a) & 0.00 & 3.23 & 4.89 & $3.19(1)$ & $2.55(10)$ & $2.48(4)$ & 3.31 \\
(b) & 0.75 & 2.46 & 3.24 & $3.27(1)$ & $2.32(20)$ & $2.59(2)$ & 2.40 \\
(c) & 0.82 & 3.12 & 5.10 & $2.89(1)$ & $2.67(14)$ & $2.73(4)$ & 4.29 \\
(d) & 0.97 & 7.97 & 6.46 & $3.48(1)$ & $2.73(14)$ & $3.10(5)$ & 6.41 \\
(e) & 1.23 & 12.23 & 12.35 & $9.04(0)$ & $2.96(6)$ & $2.51(3)$ & 10.47 \\
\hline
\end{tabular}




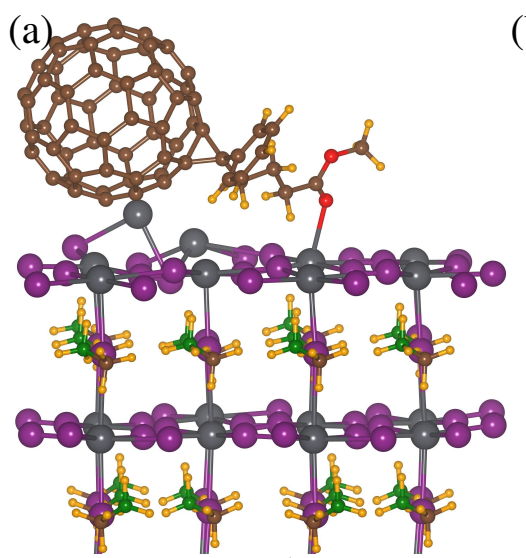

(d) (b)

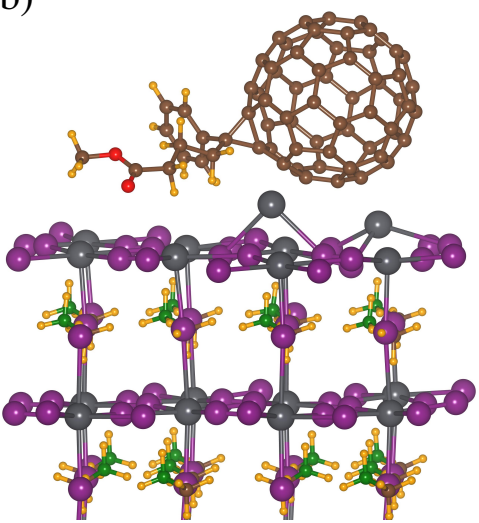

(c)

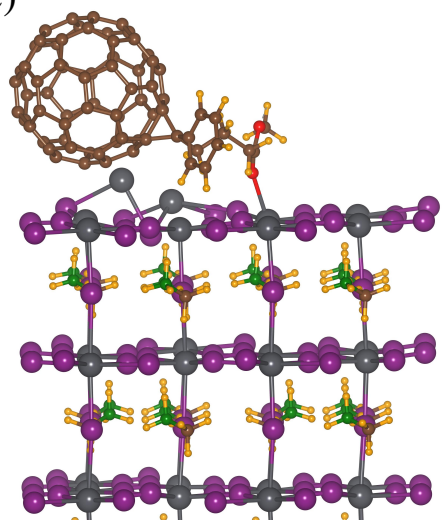

(e)

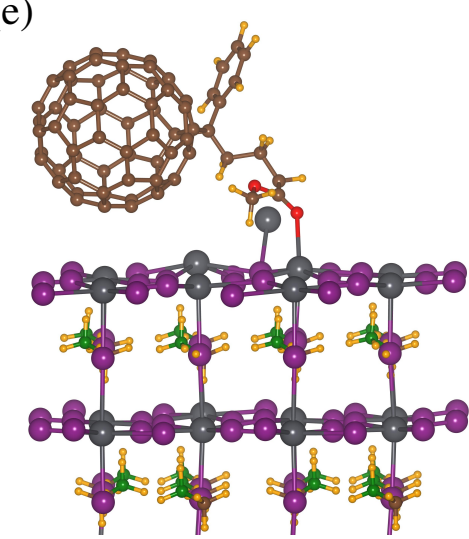

Figure S7: Atomic structure of $\mathrm{PCBM} /$ perovskite interface with $\mathrm{Pb}$ interstitial on $\mathrm{Pb}_{2}$ surface termination. Different structure corresponds to the minimum or close to minimum energy structures as shown in the Table S7.

Table S7: Perovskite-PCBM interface ( $\mathrm{PbI}_{2}$ surface termination) with $\mathrm{Pb}$ interstitials inter-atomic distances in $\AA$ for lowest five energy configurations, number in parentheses represents the total number of distances less than $3.5 \AA$.

\begin{tabular}{|l|l|l|l|l|l|l|}
\hline Position & Energy(eV) & $\mathrm{Pb}-\mathrm{O} 1$ & $\mathrm{~Pb}-\mathrm{O} 2$ & $\mathrm{I}-\mathrm{H}$ & $\mathrm{Pb}-\mathrm{C}(60)$ & $\mathrm{Pb}-\mathrm{C}(\mathrm{ester})$ \\
\hline (a) & 0.00 & 2.71 & 4.88 & $3.17(5)$ & $3.76(0)$ & 3.76 \\
(b) & 0.02 & 3.66 & 5.40 & $3.27(2)$ & $2.70(8)$ & 2.70 \\
(c) & 0.23 & 2.56 & 4.65 & $2.96(6)$ & $3.30(2)$ & - \\
(d) & 0.66 & 6.31 & 5.07 & $3.63(0)$ & $3.01(2)$ & - \\
(e) & 0.74 & 2.82 & 4.10 & $3.19(1)$ & $3.31(1)$ & 2.49 \\
\hline
\end{tabular}

